\title{
Multi-channel Coordinated Spectrum Sensing in Cognitive Radio
}

\author{
Youxuan Zhao, Bisheng Pan, Hanying Hu \\ Institute of Information Engineering, \\ The PLA Information Engineering University, \\ Zhengzhou, China, \\ zhaoyouxuan@126.com
}

\begin{abstract}
A multi-channel coordinated spectrum sensing method based on improved Genetic Algorithm is proposed to tackle low throughput issue due to inefficient signal channel serial detection in conventional cognitive radio. The proposed method detects different spatial frequency channels with multiantenna of cognitive user synchronously. Under the limitation of target detection probability, improved Genetic Algorithm is utilized for optimization of antenna allocation to maximum throughput of cognitive channel. With fully exploiting the diversity of multi-antenna, low throughput of conventional algorithm is improved in cognitive system. The simulation shows, the proposed algorithm increases throughput of cognitive channel significantly when compared with random allocation.
\end{abstract}

Keywords-cognitive radio; coordinated spectrum sensing; multi-channel; improved genetic algorithm

\section{INTRODUCTION}

Cognitive radio allows cognitive users to reuse licensed spectrum when idle, which significantly improves the spectrum efficiency [1]. Hence, determining whether licensed user is occupying the channel becomes a basic issue in cognitive radio. Single channel serial detection is normally used in conventional licensed channel detection, in which cognitive user only detects one channel every time. For the sake of improving the accuracy of single channel detection, cooperative detection, in which multiple cognitive users detects single channel simultaneously, can be adopted. However, conventional single channel detection limits detection probability greatly and thus lower probability to find spectrum opportunity [2].

A preliminary idea of coordinated spectrum detection was proposed in literature [3] to deal with the issue mentioned above. Cognitive system can obtain the whole information of occupation state in each licensed channel of cognitive spectrum band by allocating multiple cognitive terminals to detect different sub-channel. Method of random allocation which allocates the task of detecting different channels to each cognitive terminals was adopted in literature [4] and [5] based on coordinated spectrum detection. Literature [6] raised a novel parallel spectrum detection which selects several cognitive users to perform detection. Each cognitive user can detect different channel during detection period, which results in simultaneous detection of multiple channels within detection period and significant improvement of detection probability.
In the research above, cognitive user needs to transmit detection information to fusion center by control channel for final merging and determining. However, research above assume under perfect control channel condition without considering the influence of transmission error in control channel. Actually, transmission error exists even if channel coding and power control technique is used [7].

In multi-antenna cognitive radio system, cognitive user can detect multiple channels simultaneously taking advantage of spatial diversity. Cognitive user merges detection results of multiple antennas, where no control channel exists. Therefore, multi-channel coordinated spectrum detection under multi-antenna condition will be considered below. The main issues are how to allocate receive antennas to maximum throughput of cognitive channel when multiple antennas perform coordinated detection of multi-channel.

The remaining content is organized as follows. System model is given in section II. Section III descripts how to allocate antenna to maximum the total throughput of cognitive channel and proposed an optimal method based on improved Genetic Algorithm with detail description of algorithm process. Simulation verification is given in section IV which shows improved Genetic Algorithm efficiently improve system throughput.

\section{SYSTEM MODEL}

Consider such a cognitive system, which contains $\mathrm{K}$ licensed channels, one cognitive user with $\mathrm{M}$ antennas, as showed in figure 1 . Depending on the presence of licensed user in licensed channel, signal of the ith antenna from the $n$th channel can be written as follows:

$$
\begin{cases}y_{n}(t)=v_{n}(t) & H_{0, n} \\ y_{n}(t)=s_{n}(t)+v_{n}(t) & H_{1, n}\end{cases}
$$

Where, $i$ denotes antenna number from 1 to $\mathrm{M}, n$ denotes licensed channel number from 1 to $\mathrm{K}, H_{0, n}$ and $H_{1, n}$ denote hypothesis of license channel idle and occupied, respectively. Assume that received signal of licensed user $S_{n}(t)$ and noise signal $v_{n}(t)$ are independent identically distributed complex Gaussian random variables, that is $s_{n}(t) \sim \operatorname{CN}\left(0, P_{n}\right), v_{n}(t) \sim \mathcal{C N}\left(0, \sigma^{2}\right) . P_{n}$ and $\sigma^{2}$ denotes mean power of signal and noise respectively. 


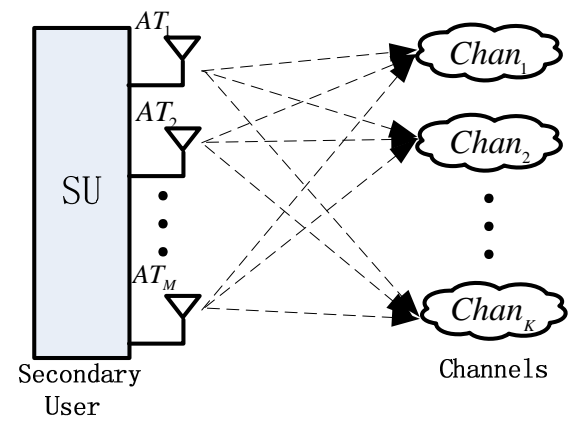

Figure 1. system model of multi-antenna multi-channel coordinated detection

\section{OPTIMIZATION OF COORDINATED SPECTRUM SENSING IN MULTIPLE CHANNELS}

\section{A. Coordinated spectrum sensing in multiple channels}

Energy detection is widely adopted recently due to simple implement, no limitation to detection signal type and convenience to carry out [8]. For the reasons above, this article select energy detection as detection method. The test statistics is expressed as follows:

$$
W_{n}=\frac{1}{N} \sum_{k=0}^{N-1} y_{n}^{2}(k)
$$

Thus, hypothesis exists according to communication state of licensed channel:

$$
H= \begin{cases}H_{0, n} & W_{n}<\lambda_{n} \\ H_{1, n} & W_{n} \geq \lambda_{n}\end{cases}
$$

Where, $\lambda_{n}$ denotes decision threshold of $n$th licensed channel. $H=H_{0, n}$ denotes $n$th channel is occupied, $H=H_{1, n}$, otherwise, denotes $n$th channel is idle. The false alarm probability and detection probability of single antenna in $n$th licensed channel is expressed as follows:

$$
\begin{gathered}
P_{f, n}=Q\left(\frac{\lambda_{n}-\sigma^{2}}{\sqrt{2 / N} \sigma^{2}}\right) \\
P_{d, n}=Q\left(\frac{\lambda_{n}-\left(P_{n}+\sigma^{2}\right)}{\sqrt{2 / N}\left(P_{n}+\sigma 2\right)}\right)
\end{gathered}
$$

Assume that the quantity of antennas used to detect channel $n$ is $T_{n}$, and the false alarm probability and detection probability is $P_{F, n}$ and $P_{D, n}$, respectively.

Define cognitive channel throughput as follows to measure performance of channel detection method.

Definition (cognitive channel throughput): cognitive channel throughput is throughput acquired by cognitive user when use idle channel to transmit data.
Let $R_{0, n}$ be transmission rate of cognitive user when licensed user of channel $n$ is absence, $R_{1, n}$ be transmission when licensed user of channel $n$ is presence, $\tau$ be channel detection time of cognitive user. Assume that the absence probability of licensed user is $P\left(H_{0, n}\right)$ and the presence probability of licensed user is $P\left(H_{1, n}\right)$. Thus, two situations exist according to the occupation of licensed channel.

i. Channel $\mathrm{n}$ is detected to be unoccupied and unoccupied actually. The probability of this case is $P\left(H_{0, n}\right)\left(1-P_{F, n}\right)$ and the throughput of this case is $\left(1-\frac{\tau}{T}\right) R_{0, n} P\left(H_{0, n}\right)\left(1-P_{F, n}\right)$.

ii. Channel $\mathrm{n}$ is detected to be unoccupied but occupied actually. The probability of this case is $P\left(H_{1, n}\right)\left(1-P_{D, n}\right)$ and the throughput of this case is $\left(1-\frac{\tau}{T}\right) R_{1, n} P\left(H_{1, n}\right)\left(1-P_{D, n}\right)$. However, in this case, cognitive user is not actually using idle channel to perform data transmission. Therefore, throughput in this case cannot be added to cognitive channel throughput.

From discussion above, cognitive channel throughput of channel $n$ is as follows:

$$
C_{n}=\left\{\begin{array}{lc}
\left(1-\frac{\tau}{T}\right) R_{0, n} P\left(H_{0, n}\right)\left(1-P_{F, n}\right), & T_{n} \neq 0 \\
0, & \text { else }
\end{array}\right.
$$

Thus, the sum of all cognitive channel throughputs is expressed as:

$$
C=\sum_{n=1}^{K} C_{n}
$$

The optimal antenna allocation should maximize the cognitive channel throughput above. The optimization issue can be descripted as: for given $K$ channels, $M$ cognitive receiving antennas and idle probability $P\left(H_{0, n}\right)$ of channel $n$, optimal target is finding $\left(T_{1}, T_{2}, \cdots T_{K}\right)$ to maximize sum of $\mathrm{N}$ cognitive channel throughputs. Besides, detection probability should be greater than a certain value $\bar{P}_{D}$ to provide adequate protection of licensed user in cognitive channel. 


$$
\begin{array}{ll}
\max _{m_{i}} & C=\sum_{n=1}^{K} C_{n} \\
\text { s.t. } & P_{D, i} \geq \bar{P}_{D} \\
& \sum_{n=1}^{K} T_{n}=M \\
& T_{n} \geq 0 \\
& T_{n} \in \mathbf{Z}, \quad \forall n \in(1, K)
\end{array}
$$

For the sake of simpleness, we assume that the detection time of each channel is a certain value $\tau$ and each channel has the same transmission rate, that is, $R_{0, n}=R_{0}$. Thus, the formula above is equal to:

$$
\begin{array}{ll}
\max _{m_{i}} & C=\sum_{n=1}^{K}\left(1-\frac{\tau}{T}\right) R_{0} P\left(H_{0, n}\right)\left(1-P_{F, n}\right) \\
\text { S.t. } & P_{D, i} \geq \bar{P}_{D} \\
& \sum_{n=1}^{K} T_{n}=M \\
& T_{n} \geq 0 \\
& T_{n} \in \mathbf{Z}, \quad \forall n \in(1, N)
\end{array}
$$

\section{B. Optimal method based on improved Genetic Algorithm}

The main target of this article is maximizing the total throughput of whole cognitive radio system by optimization of antenna allocation. As expressed in equation (9), the issue of multi-channel coordinated detection is converted to multitarget optimization issue, which can be solved by Genetic Algorithm [9].

Genetic Algorithm is an adaptive probability optimization technique based on biological genetics and evolutionism, which can provide a general framework to solve complex non-linear and non-gibbous system optimization issues. Natural selection and selection, intersection, variation in inheritance is imitated. The imitation starts from an initial group and then generates a group of more suitable individuals by operations of random selection, intersection and variation to make the group grow to better area of search space. The most suitable individual, which is the optimal solution of issue, is generated by evolution after generations.

Genetic Algorithm is widely applicable due to its convenience, low computational cost, fast operation, simple implementation and few adjustable parameters [10]. However, research about Genetic Algorithm shows that algorithm with strong search capability tend to early convergence, which result in local optimal solution and miss the best search result. When early convergence appears, much computation will be ruined on development which can hardly progress after convergence. If the capability of Genetic Algorithm to get rid of early convergence is weak, the solution of multi-target optimization is difficult to handle.
Hence, it necessitates improved variation operation of Genetic Algorithm to enhance the variety and becomes a main key point of multi-target optimization in Genetic Algorithm.

The improved Genetic Algorithm proposed in this article, that is distributed Genetic Algorithm, has the characteristic of multi-target optimization application. Single group in conventional Genetic Algorithm is divided into a target group and several subgroups, which develop independent. By the information exchange between subgroups and target group, variety of target group is enhanced. The schematic structure of distributed Genetic Algorithm is illustrated in figure 2.

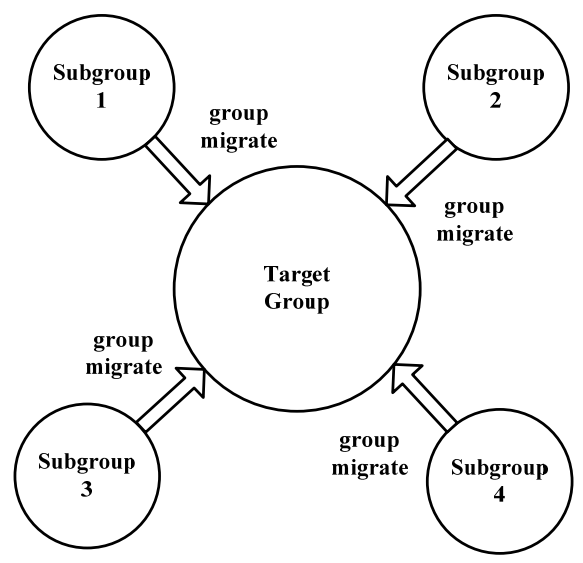

Figure 2. Schematic structure of distributed Gentic Algorithm

The distributed Genetic Algorithm changes the evolution mechanism in conventional Genetic Algorithm from evolution of single group to cooperative evolution between several small groups. The variety of target group is enhanced to get rid of early convergence. The steps of applying improved Genetic Algorithm to solve coordinated multichannel detection is as follows.

Step1: Initialization. Generate P subgroups of which each contains Q individuals randomly. Generate a marrow group which contains $\mathrm{P}$ individuals randomly.

Step2: Assessment. Calculate the suitability value of each individual according to target function.

Step3: Intersection. Select a certain amount of individuals within each subgroup according to intersection probability to make pairs and choose intersection point randomly to intersect. When selecting individuals, the greater suitability the higher intersection probability.

Step4: Variation. Select part of individuals from those after intersection within each group according to a certain variation probability for variation.

Step5: Migration. Search individual with highest suitability in each group to compare its suitability value with suitability values of individuals in marrow group. If individual in subgroup has greater suitability value than a certain individual in marrow group, move this individual in subgroup to marrow group to replace the individual with lower suitability value in marrow group. 
Step6: Termination. Repeat step2 to step5 until the suitability values of individuals in marrow group keep the same after a certain times of iteration.

Step7: Output. Output the optimal solution and calculate the optimal channel capacity.

\section{SIMULATION RESULTS AND PERFORMANCE ANALYSIS}

This section simulates the performance of multi-channel coordinated detection. Assume that quantity of cognitive user antennas is $M=20$, quantity of channel for detection is $5,10,15$, idle probability of licensed channel is $P\left(H_{0, n}\right)=0.7$

and detection probability is $P_{D} \geq 0.99$.Cognitive channel throughput of coordinated detection using optimal antenna allocation method, random antenna allocation method and allocation method based on improved Genetic Algorithm is analyzed.

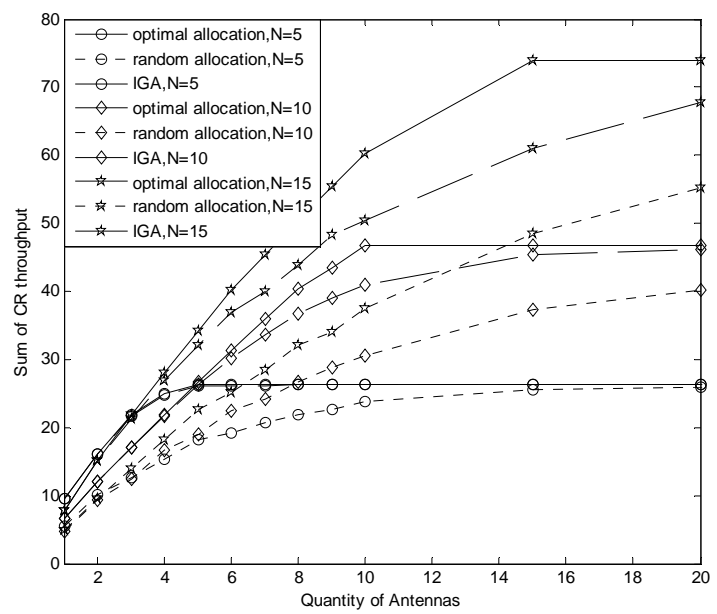

Figure 3. Cognitive channel throughput performance of different allocation methods under AND criterion

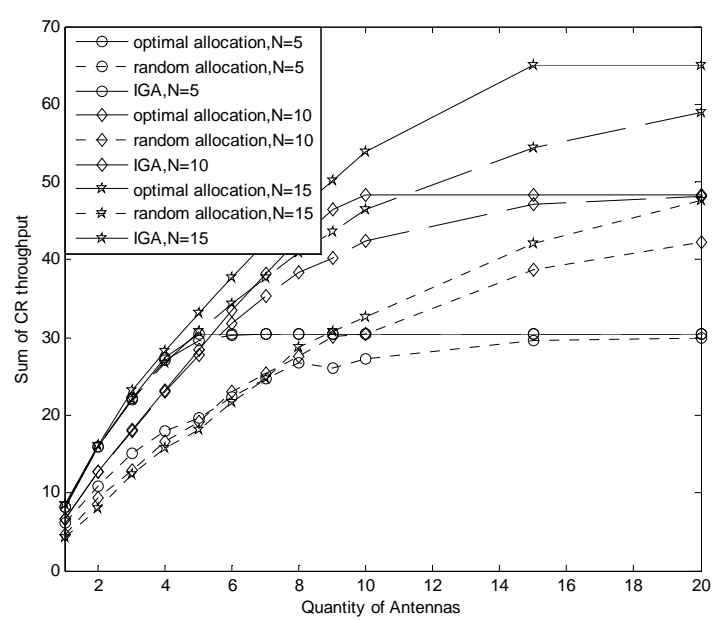

Figure 4. Cognitive channel throughput performance of different allocation methods under OR criterion
Figure 3 and figure 4 shows cognitive channel total throughput of parallel detection using different allocation methods under AND criterion and OR criterion respectively. From the figures, we can get

1) The tendency of performance curve agreed between AND criterion and OR criterion. It is clear that proposed coordinated detection method based on improved Genetic Algorithm is close to optimal algorithm, surpassing cognitive channel total throughput obtained by random allocation method.

2) Cognitive channel total throughput of multiple channles increases as the quantity of channels increases, which coincide with our intuition. More available channels due to increase of channel quantity results in increase of cognitive channel total throughput.

3) Cognitive channel total throughput increases as the quantity of antennas inceases. Performance of coordinated detection is improved due to quantity increase of antenna for coordinated detection in each channel. Thus, cognitive channel throughput of each channel is improved and cognitive channel total throughput is improved therefore.

4) There exists a turning point on the curve of cognitive channel total throughput varing with quntity increase of cognitive user antenna. When quantity of channels is greater than quantity of antennas, cognitive channel throughput increases rapidly with the quantity increase of antenna. Since the quantity increase of antenna means the increase of channel quantity in parallel detection, therefore cognitive channel total throughput rapidly increase due to utilization of more available channels. When quantity of antenna increases to greater than quantity of channels, performance of cognitive channel throughput increases slow. Since in this case cognitive channel total throughput increases due to the increase of detection performance in each channel which is relatively slow.

\section{CONCLUSION}

This article discusses optimization issue of the multiantenna multi-channel coordinated detection, utilizing antenna allocation based on the improved Genetic Algorithm. Multi-antenna coordinated spectrum detection is not affected by control channel, solving the problem of transmission error in control channel of conventional coordinated detection. The improved Genetic Algorithm varies the evolution mechanism of conventional Genetic Algorithm, and aims at enhancing the variety of target group to avoid early convergence. Simulation results shows, the proposed algorithm can significantly raise the cognitive channel total throughput, surpassing the random allocation method.

\section{REFERENCES}

[1] Z. L. Wang, Z. Y. Feng and P. Zhang, "An Iterative Hungarian Algorithm Based Coordinated Spectrum Sensing Strategy,” IEEE Communications Letters, vol. 15, no. 1, pp. 49-41. Jan. 2011. 
[2] H. KIM and K. G. SHIN, "Efficient discovery of spectrum opportunities with MAC-layer sensing in cognitive radio networks," IEEE Transactions on Mobile Computing, vol. 7, no. 5, pp. 533-545. May 2008.

[3] C. H. Lee and W. Wolf, "Multiple access-inspired cooperative spectrum sensing for cognitive radio," IEEE Military Communications Conference, Orlando, FL, Oct.2007.

[4] W. Zhang, R. K. Mallik and K. Ben Lataief, "Cooperative Shared Spectrum Sensing for Dynamic Cognitive Radio Networks,” Proc. IEEE International Conference on Communications 2008(ICC'08), Beijing, China. pp. 19-23. May 2008.

[5] P. Y. Wang, Z. Y. Zhang, H. Huang, K. D. Wu, G. D, Yu and A. P. Huang, "Multi-channel Cooperative Spectrum Sensing Based on Belief Propagation Algorithm", Proc. IEEE $70^{\text {th }}$ Vehicular Technology Conference (VTC2009-Fall), Anchorage, USA, Sept. pp. $20-23,2009$
[6] S. L. Xie and Y. Liu, "A Parallel Cooperative Spectrum Sensing in Cognitive Radio Networks," IEEE Transactions on Vehicular Technology, vol. 59, no. 8, pp. 4079-4092. Oct. 2010.

[7] C. LEE, W. Wolf, "Energy efficient techniques for cooperative spectrum sensing in cognitive radios,” IEEE CCNC. Las Ve-gas. pp. 968-972. 2008.

[8] F. F. Digham, M. K. Simon, "On the Energy Detection of Unknown Signals over Fading Channels," IEEE Transaction on Wireless Communications, vol. 55, no. 1, pp. 21-24. 2007.

[9] C. A. COELLO, G. B.LAMONT, A. V. VELDHUIZEN, "Evolutionary Algorithms for Solving Multi-Objective Problems," 2nd ed, New York: Springer-Verlag, 2007.

[10] X. M. HU, J. ZHANG, Y. YU, "Hybrid genetic algorithm using a forward encoding scheme for lifetime maximization of wireless sensor networks," IEEE Trans on Evol Comp, vol. 14, no. 5. pp. 766781.2010. 